\title{
Transversus abdominis plane block reduces remifentanil and propofol consumption, evaluated by closed-loop titration guided by bispectral index
}

\author{
JIAHAI MA ${ }^{1,2^{*}}$, XUE-YAN WANG ${ }^{3 *}$, QIAO-XIA SUN ${ }^{1}$, JON ZHOU $^{2}$, TAO LI $^{1}$, \\ MEI-RU JIANG ${ }^{1}$, GANG-GANG LIU ${ }^{1}$ and HONG LIU ${ }^{2}$
}

\begin{abstract}
${ }^{1}$ Department of Anesthesiology, The Affiliated Yantai Yuhuangding Hospital of Qingdao University, Yantai, Shandong 264000, P.R. China; ${ }^{2}$ Department of Anesthesiology and Pain Medicine, University of California Davis Health, Sacramento, CA 95817, USA; ${ }^{3}$ Yantai Center of Disease Control and Prevention, Yantai, Shandong 264003, P.R. China
\end{abstract}

Received March 7, 2018; Accepted August 9, 2018

DOI: $10.3892 /$ etm.2018.6707

\begin{abstract}
The present prospective, randomized, double-blind study aimed to determine the impact of transversus abdominis plane (TAP) block on propofol and remifentanil consumption, when administered by closed-loop titration guided by processed electroencephalography, i.e., bispectral index (BIS) values. Following institutional review board approval, 60 patients were scheduled for laparoscopic colectomy under general anesthesia. Patients were randomly assigned to receive bilateral TAP block with $20 \mathrm{ml} 0.375 \%$ ropivacaine (TAP group) or $20 \mathrm{ml} \mathrm{0.9 \%}$ saline [control (CON) group]. General anesthesia was maintained with propofol and remifentanil administration using closed-loop titration guided by BIS values. The primary outcome was perioperative propofol and remifentanil consumption. The secondary outcomes were hypertensive or hypotensive events requiring treatment, recovery time in PACU and time to first rescue analgesia following surgery. A total of 58 patients participated in the present study. At similar depths of anesthesia, as measured by BIS during the maintenance phase (45-55), patients who received TAP blocks required less propofol $(4.2 \pm 1.3$ vs. $5.5 \pm 1.6 \mathrm{mg} / \mathrm{kg} / \mathrm{h} ; \mathrm{P}<0.001)$ and remifentanil $(0.16 \pm 0.05$ vs. $0.21 \pm 0.05 \mu \mathrm{g} / \mathrm{kg} / \mathrm{min} ; \mathrm{P}<0.001)$. Time to extubation was significantly shorter in the TAP group $(9.8 \pm 3.2 \mathrm{~min})$
\end{abstract}

Correspondence to: Dr Jiahai Ma, Department of Anesthesiology, The Affiliated Yantai Yuhuangding Hospital of Qingdao University, 20 Yuhuangding East Road, Yantai, Shandong 264000, P.R. China E-mail:mjh-214@163.com

Dr Hong Liu, Department of Anesthesiology and Pain Medicine, University of California Davis Health, 4150 V Street Suite 1200, Sacramento, CA 95817, USA

E-mail: hualiu@ucdavis.edu

*Contributed equally

Key words: transversus abdominis plane block, remifentanil, propofol, closed-loop titration, laparoscopic colectomy than in the CON group $(14.2 \pm 4.9 \mathrm{~min})(\mathrm{P}<0.05)$. The requirement to treat hemodynamic change was also significantly lower $(\mathrm{P}<0.05)$. Pain score at $2 \mathrm{~h}$ after surgery was also significantly reduced in the TAP group compared with the CON group $(\mathrm{P}<0.05)$, whereas the time to first rescue analgesia was delayed in patients who received TAP block $(\mathrm{P}<0.05)$. Postoperative nausea and vomiting occurred at comparable rates in each group (P>0.05). In conclusion, TAP block combined with general anesthesia reduced propofol and remifentanil consumption, shortened time to tracheal extubation and promoted hemodynamic stability in laparoscopic colectomy.

\section{Introduction}

The concept of enhanced recovery after surgery (ERAS) has been widely accepted in clinical practice, with the benefits of improving outcomes and decreasing length of hospital stay (1). The important components of a successful anesthetic in an ERAS protocol are reduction of opioid use and optimized pain control using a multimodal approach, which includes neuroaxial or regional anesthesia techniques for the primary anesthetic (2). Transversus abdominis plane (TAP) block is a regional anesthesia technique that has been widely used in abdominal surgery $(3,4)$. TAP block provides effective postoperative analgesia following abdominal surgery, and it can be a useful strategy to reduce perioperative opioid consumption, maintain intraoperative hemodynamic stability and promote early recovery from anesthesia $(5,6)$. In addition, it is simple to perform TAP block, and there are minimal procedure-associated complications (7).

Although numerous studies have been performed regarding the impact of TAP blocks on postoperative analgesia (4-6), the usefulness of TAP blocks during surgery have not been well studied. As an important part of a multimodal analgesic regimen, TAP blocks can reduce intraoperative opioid use. Recently, closed-loop titration guided by processed electroencephalography, such as bispectral index (BIS), has become clinically applicable, and can result in improved anesthetic control (8). The system automates anesthetic administration and thus helps avoid human error $(8,9)$. 
This automated system may enable quantification of the anesthetic-sparing effect of an adjuvant, such as epidural catheter or TAP block for analgesia $(8,9)$. A recent study indicated that thoracic epidural analgesia combined with general anesthesia could decrease remifentanil and propofol requirements by one-half and one-third when using hemodynamic criteria or BIS for the titration of analgesia, respectively (9).

The present study was designed to use a closed-loop titration system guided by BIS to quantify the effect of TAP blocks on the intraoperative use of propofol and remifentanil. The primary hypothesis was that TAP blocks may decrease perioperative propofol and remifentanil consumption under general anesthesia. Secondly, the effect of TAP blocks on hemodynamics, time to tracheal extubation and postoperative analgesia were investigated.

\section{Materials and methods}

Study protocol. This prospective, randomized, double-blind clinical study was approved by the local ethics committee clinical trials register (approval no. ChiCTR-IOR-17014 111). Following the collection of written informed consent, 60 patients aged 18-75 years with American Society of Anesthesiologists physical status score II/III $(3,6)$ scheduled for laparoscopic colectomy under general anesthesia were enrolled in the present study. Patients were recruited from March 1st 2017 to February 25th 2018 in Yantai Yuhuangding Hospital (Yantai, China).

Randomization and blinding. Patients were subjected to the following exclusion criteria: History of allergy to the drugs used in the present study, psychological disorders, infection of the block site, any other contraindications of TAP block, tolerance to opioids or use of opioids within $48 \mathrm{~h}$ prior to the study. The patients and all staff involved in patient management and data collection were blinded to group assignment until the end of the study. All TAP blocks were performed by experienced anesthesiologists who did not participate in data collection. The bilateral TAP blocks were performed under general anesthesia. A third anesthesiologist, who was not involved in the management of the patients or study, prepared the randomized medications. The prepared syringes contained either $20 \mathrm{ml}$ normal saline for the CON group or $20 \mathrm{ml} 0.375 \%$ ropivacaine (Qilu Pharmaceutical Co., Ltd., Jinan, China) for the TAP group.

Anesthesia and TAP block. The visual analogue scale (VAS) $(0=$ no pain and $10=$ the worst possible pain) was used for postoperative pain assessment. On arrival at the operating room, patients were randomized into the control (CON) group $(n=30)$ or the TAP group $(n=30)$ using a computer-produced randomized list. All patients were non-invasively monitored for blood pressure (BP), electrocardiogram (ECG), heart rate (HR) and oxygen saturation $\left(\mathrm{SpO}_{2}\right)$. Neuromuscular blockade was monitored using the CONCERT-CL neuromuscular blockade monitoring system provided by VERTARK Technology Co., Ltd. (Guangxi, China), whereas BIS was monitored using a BIS $^{\text {тм }}$ (Aspect Medical Systems, Inc., Norwood, MA, USA). General anesthesia was induced using $0.05 \mathrm{mg} / \mathrm{kg}$ midazolam (Jiangsu Nhwa Pharmaceutical Co., Ltd. Xuzhou, China),
$0.4 \mu \mathrm{g} / \mathrm{kg}$ sufentanil (Yichang Humanwell Pharmaceutical Co., Ltd., Yichang China), 1.5-2.0 mg/kg propofol (Diprivan; AstraZeneca, Macclesfield, UK) and $0.15 \mathrm{mg} / \mathrm{kg}$ cisatracurium (Jiangsu Hengrui Medicine Co., Ltd, Jiangsu, China). Following intubation, general anesthesia was maintained by propofol and remifentanil using closed-loop titration guided by BIS. No inhalation anesthetic was used. A previously described titration system and parameters of target-controlled infusion were used for propofol and remifentanil (Yichang Humanwell, Pharmaceutical Co., Ltd., Yichang, China), respectively (10-12). The initial target concentrations of propofol in the plasma $(2-4 \mu \mathrm{g} / \mathrm{ml})$ were selected by the anesthesiologists according to standard practice. The target concentration of propofol was automatically adjusted using the closed-loop system to maintain a BIS value between 45-55 during surgery. The target concentration of remifentanil was set at $2-8 \mathrm{ng} / \mathrm{ml}$. The anesthesiologists administered the medication manually or switched to manual infusion during surgery if required. The closed-loop infusion of cisatracurium was used following induction guided by the reappearance of the second twitch during train of four stimulation. Ringer's acetate solution was used for volume replacement. All aspects of anesthesia management, except for the drug infusion, were performed by the anesthesiologists according to current clinical practice.

The tidal volume was set at $6-8 \mathrm{ml} / \mathrm{kg}$ and the respiratory rate at 12 breaths/min to maintain end-tidal $\mathrm{CO}_{2}$ tension at $35-45 \mathrm{mmHg}$. Sufentanil was administered at $0.3 \mu \mathrm{g} / \mathrm{kg}$ prior to skin incision. TAP block was performed following induction using a similar method to that previously described (13). Briefly, a high-frequency linear ultrasound (US) probe (Venue 50; 7-12 MHz; GE Healthcare, Chicago, IL, USA) was placed laterally to the abdominal wall between the costal margin and the iliac crest on the mid axillary line. A satisfactory image aimed to visualize the subcutaneous fat, external oblique muscle, internal oblique muscle, transversus abdominis muscle, peritoneum and intraperitoneal cavity. The needle was introduced in the plane of the ultrasound probe directly beneath the probe and advanced until it reached the plane between the internal oblique and the transversus abdominis muscles. The correct location of the needle tip was confirmed by injection of $1 \mathrm{ml}$ normal saline, which resulted in separation between the internal oblique and transversus abdominis muscle. Subsequently, $20 \mathrm{ml}$ prepared solution was injected under US-directed visualization. The same steps were repeated on the opposite side.

Hemodynamic changes were treated according to study protocol. Atropine (Shandong Xinhua Pharmaceutical Co., Ltd., Zibo, China) was administered when HR was $<45$ bpm, esmolol (Qilu Pharmaceutical Co., Ltd.) was administered when HR exceeded $120 \mathrm{bpm}$, phenylephrine was administered when BP was $<80 \%$ of the pre-induction value, and nicardipine was administered when systolic blood pressure exceeded $160 \mathrm{mmHg}$.

A total of $5 \mathrm{mg}$ dezocine (Yangtze River Pharmaceutical Group, Taizhou, China) and $0.25 \mathrm{mg}$ palonosetron (Qilu Pharmaceutical Co., Ltd.) were administered intravenously (IV) $30 \mathrm{~min}$ prior to the end of surgery. The infusion of cisatracurium was stopped $\sim 30$ min prior to the end of surgery. Upon completion of surgery, patients were treated with $1.0 \mathrm{mg}$ neostigmine (Shandong Xinhua Pharmaceutical Group Co., Ltd., Zibo, China) and $0.5 \mathrm{mg}$ atropine, if necessary, to achieve 


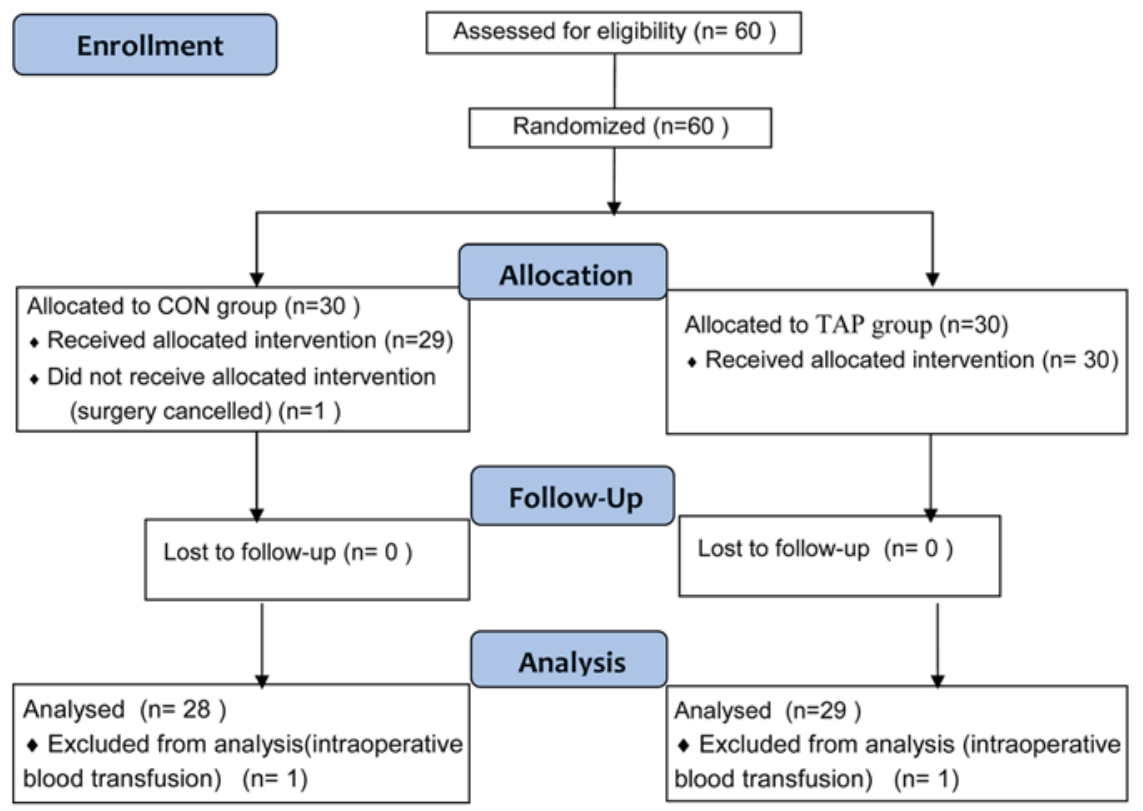

Figure 1. CONSORT flow diagram. CON, control; TAP, transversus abdominis plane.

$\mathrm{a} \mathrm{T}_{4} / \mathrm{T}_{1}$ ratio $>0.9$, and the propofol and remifentanil infusions were stopped. Patients were extubated once the extubation criteria were met, and time to extubation was recorded. The patients were transferred to the post-anesthesia care unit (PACU) where they received nasal $\mathrm{O}_{2}$ supplementation and were monitored continuously for vital signs (HR, BP, ECG and $\mathrm{SpO}_{2}$ ). Complications were recorded for $6 \mathrm{~h}$ postoperatively, including nausea, vomiting, hemodynamic instability, desaturation or apnea. The VAS score at $2 \mathrm{~h}$ post-surgery and the time to the first rescue analgesic request were recorded. A total of $5 \mathrm{mg}$ dezocine IV was administered as rescue analgesia when the VAS score was $\geq 4$. A total of $4 \mathrm{mg}$ ondansetron (Qilu Pharmaceutical Co., Ltd.) was administered IV if necessary to relieve nausea or vomiting.

Statistical analysis. The mean propofol dosage required for maintenance of anesthesia was $4.7 \pm 1.6 \mathrm{mg} / \mathrm{kg} / \mathrm{h}$ when propofol and remifentanil were co-administered via closed-loop titration guided by BIS (14). The present study was designed to provide $90 \%$ power for detecting a $30 \%$ decrease in propofol dosage among patients receiving TAP block with a bilateral $\alpha$ risk value of 0.05 . A total of 60 patients were recruited in the present study. All data in the present study were analyzed with SPSS (version 17.0; SPSS, Inc., Armonk, NY, USA). Data were presented as the mean \pm standard deviation and count $(\%)$, as appropriate. Following assessment of normality, continuous data were compared using Student's t-test, whereas the Mann-Whitney test was performed to compare non-continuous and non-normally distributed data. $\chi^{2}$ or Fisher's exact tests were used to analyze proportions. $\mathrm{P}<0.05$ was considered to indicate a statistically significant difference.

\section{Results}

Fig. 1 illustrates the CONSORT flow diagram of patient recruitment. A total of 60 patients were enrolled in the present trial. In the CON group, 1 patient withdrew due to surgery cancellation, and a further 2 patients ( 1 from the CON group and 1 from the TAP group) were excluded due to intraoperative blood transfusion. A total of 57 patients completed the trial (28 in the CON group and 29 in the TAP group). Patient characteristics did not differ significantly between the two groups $(\mathrm{P}>0.05)$. Demographic and surgical characteristics were comparable and there were no significant differences between the groups (Table I). The TAP block was easily localized via US and blocks were uneventfully performed in all patients. There were no complications associated with TAP block in the present study.

Similar depths of anesthesia (BIS number, 45-55) were maintained during the surgery, and the total doses of propofol and remifentanil used were significantly less in the TAP group compared with the CON group [propofol, $4.2 \pm 1.3$ vs. $5.5 \pm 1.6 \mathrm{mg} / \mathrm{kg} / \mathrm{h} \quad(\mathrm{P}<0.001)$; remifentanil, $0.16 \pm 0.05$ vs. $0.21 \pm 0.05 \mu \mathrm{g} / \mathrm{kg} / \mathrm{min}(\mathrm{P}<0.001)]$. The dose of cisatracurium was not significantly different between the two groups $(\mathrm{P}>0.05)$. The frequency of undesirable hemodynamic treatment effects (including hypotension, hypertension, bradycardia and tachycardia) was also significantly lower in the TAP group than in CON group $(\mathrm{P}<0.05$; Table II).

All patients met the extubation criteria at the end of surgery. However, time to tracheal extubation was significantly shorter in the TAP group $(9.8 \pm 3.2 \mathrm{~min})$ than the CON group $(14.2 \pm 4.9 \mathrm{~min} ; \mathrm{P}<0.05)$. VAS pain score at $2 \mathrm{~h}$ following surgery was significantly reduced in the TAP group compared with the CON group $(\mathrm{P}<0.05$; Table II). The time to first rescue analgesia was significantly delayed in the TAP group (324 $\pm 72 \mathrm{~min})$ compared with the CON group (126 $\pm 34 \mathrm{~min}$; $\mathrm{P}<0.05)$. Postoperative nausea and vomiting occurred at comparable rates in each group $(\mathrm{P}>0.05)$.

\section{Discussion}

The results of the present study indicate that TAP blocks may reduce the consumption of propofol by $28 \%$ and remifentanil 
Table I. Clinical characteristics of patients in the two groups.

\begin{tabular}{lccc}
\hline Characteristic & CON group $(\mathrm{n}=28)$ & TAP group $(\mathrm{n}=29)$ & P-value \\
\hline Age (years) & $58.5 \pm 11.2$ & $59.2 \pm 10.6$ & 0.73 \\
Male/female & $17 / 11$ & $18 / 11$ & 0.92 \\
ASA grade II/III & $10 / 18$ & $12 / 17$ & 0.66 \\
Right/left hemicoloctomy & $13 / 15$ & $15 / 14$ & 0.69 \\
Weight $(\mathrm{kg})$ & $73.2 \pm 11.4$ & $74.3 \pm 11.5$ & 0.72 \\
Height $(\mathrm{cm})$ & $168.2 \pm 6.1$ & $165.9 \pm 5.8$ & 0.15 \\
BMI $\left(\mathrm{kg} / \mathrm{m}^{2}\right)$ & $22.3 \pm 3.8$ & $22.6 \pm 4.0$ & 0.77 \\
Operative time (min) & $162.6 \pm 48.5$ & $166.8 \pm 38.7$ & 0.72 \\
Blood loss (ml) & $156 \pm 85$ & $162 \pm 94$ & 0.80 \\
Infusion volume (ml) & $1,864 \pm 425$ & $1,786 \pm 501$ & $674 \pm 121$ \\
Urine output (ml) & $624 \pm 104$ & 0.53 & 0.10 \\
\hline
\end{tabular}

Categorical variables are presented as $\mathrm{n} / \mathrm{n}$. Numerical variables are presented as mean \pm standard deviation. CON, control; TAP, transversus abdominis plane; ASA, American Society of Anesthesiologists physical status score; BMI, body mass index.

Table II. Comparison of anesthetic procedures between the two groups during the maintenance phase.

\begin{tabular}{lcc}
\hline Procedure & CON group $(\mathrm{n}=28)$ & TAP group (n=29) \\
\hline Propofol & & \\
Mean dose $(\mathrm{mg} / \mathrm{kg} / \mathrm{h})$ & $5.5 \pm 1.6$ & $4.2 \pm 1.3$ \\
Mean target concentration $(\mu \mathrm{g} / \mathrm{ml})$ & $2.7 \pm 0.7$ & $2.1 \pm 0.5$ \\
Remifentanil & & $<0.001$ \\
Mean dose $(\mu \mathrm{g} / \mathrm{kg} / \mathrm{min})$ & $0.21 \pm 0.05$ & $0.16 \pm 0.05$ \\
Mean target concentration $(\mathrm{ng} / \mathrm{ml})$ & $6.1 \pm 1.5$ & $4.8 \pm 1.1$ \\
Mean dose of cisatracurium $(\mathrm{mg} / \mathrm{kg} / \mathrm{h})$ & $0.86 \pm 0.21$ & $0.84 \pm 0.18$ \\
Undesirable hemodynamic effects & & 60.001 \\
Hypotension requiring treatment by phenylephrine & $11(39.3)$ & $20.7)$ \\
Hypertension requiring treatment by nicardipine & $7(25.0)$ & $2(7.9)$ \\
Bradycardia requiring treatment by atropine & $3(10.7)$ & $4(13.7)$ \\
Tachycardia requiring treatment by esmolol & $3(10.7)$ & $3(10.3)$ \\
Time to tracheal extubation (min) & $14.2 \pm 4.9$ & 0.914 \\
Pain score at 2 h following surgery & $3.4 \pm 1.6$ & $9.0 \pm 3.2$ \\
Time to first rescue analgesia (min) & $126 \pm 34$ & $1.8 \pm 0.6$ \\
Nausea and vomiting & $5(17.8)$ & $324 \pm 72$ \\
\end{tabular}

Continuous variables are presented as mean \pm standard deviation. Categorical variables are presented as n (\%). CON, control; TAP, transversus abdominis plane; time to tracheal extubation, time from the end of surgery to tracheal extubation.

by $26 \%$. Infusion with guided closed-loop titration promoted hemodynamic stability, shortened recovery time and improved postoperative analgesia.

The goal of anesthesia is to reduce acute postoperative pain and decrease surgical stress responses. Anesthesiologists serve an important role in facilitating the recovery of patients undergoing surgery using an ERAS protocol. Successful implementation of ERAS programs require anesthesiologists to be more involved in perioperative care and to be more aware of the impact of anesthetic techniques on surgical outcomes and recovery (15). Optimizing perioperative pain management while reducing opioid use were the major goals of the present study.
Although total intravenous anesthesia (TIVA) with propofol and remifentanil may be sufficient, prolonged use of TIVA may increase adverse effects, including delayed awakening, myocardial depression and hemodynamic instability (15). Patients undergoing abdominal surgery often suffer from uncontrolled postoperative pain $(2,3,6)$. Opioids have been used to treat moderate to severe postoperative pain; however, they may cause complications such as nausea and vomiting, respiratory depression and circulatory depression $(16,17)$. TAP block is a regional anesthetic technique that blocks the abdominal wall neural afferents by introducing local anesthetic into the fascial plane between the internal oblique 
and transversus abdominis muscles (3). Following its initial description in 2001 by Rafi et al (18), its use in postoperative pain management has gained popularity. TAP block can provide effective analgesia for a variety of abdominal surgical procedures by decreasing somatic pain (19-21). Previously, it was demonstrated that TAP blocks may decrease postoperative pain and opioid consumption in women undergoing cesarean section (22). TAP block has also been demonstrated to reduce the use of opioids, improve pain control and expedite recovery in laparoscopic colorectal surgery patients (23). A previous meta-analysis of the efficacy of TAP block demonstrated that it reduced the requirement for postoperative opioids and associated side-effects, and provided more effective pain relief (24), suggesting that there may be a role for TAP blocks as part of an effective multimodal pain regimen. Erdogan et al (5) demonstrated that there was significantly lower remifentanil consumption and anesthesia recovery time, but no significant differences in desflurane consumption in liver transplant donors who received TAP block, compared with donors who didn't receive TAP block. Tsuchiya et al (6) reported a similar sparing-effect of TAP block on sevoflurane and fentanyl use in abdominal surgery. In these studies, the titration of different anesthetics was mainly dependent on clinical hemodynamic criteria. However, hemodynamic changes are influenced by numerous variables, including preload state, pre-existing cardiovascular conditions, surgical blood loss and vasopressor usage. In the present study, anesthetic titration was performed with a closed loop automated system, limiting investigator bias. In addition, to the best of our knowledge, no previous studies quantified the opioid sparing effect of TAP blocks on propofol and remifentanil.

Anesthesiologists typically titrate anesthetics based on a combination of hemodynamic changes and clinical signs, alongside their knowledge of pharmacokinetics and pharmacodynamics. The factors involved in the decision-making process are also subject to variations between individuals. By contrast, closed-loop titration used in the present study responds to changes in BIS values, which may result in a more objective mode of maintenance of anesthesia. By adding TAP blocks to a closed-loop protocol, distinguishing the effect of propofol and remifentanil on the depth of anesthesia may be possible. Guignard et al (25) reported in a previous controlled trial that, following a pain stimulus, the BIS and hemodynamics changes were similar. TAP blocks demonstrated a sparing effect on the amount of propofol and remifentanil required to maintain surgical anesthesia. TAP blocks also provided additional analgesia by delaying the time for first rescue analgesic request, and VAS pain scores $2 \mathrm{~h}$ after surgery were lower, which is consistent with past meta-analysis studies $(19,22,23)$.

Although intraoperative safety of surgical patients has greatly improved, cardiovascular complications still pose significant risks to high-risk patients with severe cardiovascular disease undergoing abdominal surgery (26). In the present study, patients who received TAP blocks exhibited less significant changes in hemodynamics that required treatment with vasopressors. These findings may be beneficial for critically ill and elderly patients who may be more vulnerable to changes in hemodynamics.

The time to tracheal extubation was also decreased in patients with TAP blocks, likely attributable to a decrease in total remifentanil and propofol levels. The incidence of postoperative nausea and vomiting was similar in both groups. However, the present study was not powered for this result, given the low incidence in patients who received total IV anesthesia and palonosetron prior to the end of surgery.

There are limitations of the present study. Although VAS scores were lower in the TAP block group, there is no objective way to evaluate the anti-nociceptive effect of TAP block. Secondly, only patients aged from 18-75 years were included, whereas elderly patients ( $>75$ years) were not included, which may introduce a selection bias. A more age comprehensive study should be performed in the future. Finally, the impact of TAP blocks on the postoperative period, such as the length of stay in the PACU and hospital admission, were not investigated in the present study.

In conclusion, in laparoscopic colectomy, TAP blocks may reduce propofol and remifentanil consumption within a closed-loop anesthetic delivery system, shorten time to tracheal extubation and promote intraoperative hemodynamic stability. TAP blocks may also serve a vital role in multimodal analgesia for abdominal surgeries and promote enhanced recovery following surgery.

\section{Acknowledgements}

Not applicable.

\section{Funding}

The present study was supported by the Nature and Science Fund of Shandong Province, China (grant no. ZR2014HL109), the Science and Technology Program Foundation of Yantai, China (grant no. 2014WS009), and the Departments of Anesthesiology and Pain Medicine of University of California Davis Health (grant no. UL1 TR001860).

\section{Availabilty of data and materials}

The data used and analyzed during the current study are available from the corresponding author on reasonable request.

\section{Authors' contributions}

JM and XW performed the study and also were major contributors in writing the manuscript. QS and TL analyzed the data. JM and HL designed the study and revised the manuscript. JZ interpreted the data and revised the manuscript critically. MJ and GL performed the study and collected the data. All authors read and approved the final manuscript.

\section{Ethics approval and consent to participate}

Approval was obtained from the Institutional Human Investigations Committee and Institutional Review Board of The Affiliated Yantai Yuhuangding Hospital of Qingdao University. All patients provided written informed consent for participation.

\section{Patient consent for publication}

Not applicable. 


\section{Competing interests}

The authors declare that they have no competing interests.

\section{References}

1. Li Z, Wang Q, Li B, Bai B and Zhao Q: Influence of enhanced recovery after surgery programs on laparoscopy-assisted gastrectomy for gastric cancer: A systematic review and meta-analysis of randomized control trials. World J Surg Oncol 15: 207, 2017.

2. Wick EC, Grant MC and Wu CL: Postoperative multimoda analgesia pain management with nonopioid analgesics and techniques: A review. JAMA Surg 152: 691-697, 2017.

3. McDonnell JG, O'Donnell B, Curley G, Heffernan A, Power C and Laffey JG: The analgesic efficacy of transversus abdominis plane block after abdominal surgery: A prospective randomized controlled trial. Anesth Analg 104: 193-197, 2007.

4. Kim AJ, Yong RJ and Urman RD: The role of transversus abdominis plane blocks in enhanced recovery after surgery pathways for open and laparoscopic colorectal surgery. J Laparoendosc Adv Surg Tech A 27: 909-914, 2017.

5. Erdogan MA, Ozgul U, Uçar M, Yalin MR, Colak YZ, Çolak C and Toprak HI: Effect of transversus abdominis plane block in combination with general anesthesia on perioperative opioid consumption, hemodynamics, and recovery in living liver donors: The prospective, double-blinded, randomized study. Clin Transplant 31, 2017.

6. Tsuchiya M, Takahashi R, Furukawa A, Suehiro K, Mizutani K and Nishikawa K: Transversus abdominis plane block in combination with general anesthesia provides better intraoperative hemodynamic control and quicker recovery than general anesthesia alone in high-risk abdominal surgery patients. Minerva Anestesiol 78: 1241-1247, 2012.

7. Conaghan P, Maxwell-Armstrong C, Bedforth N, Gornal C, Baxendale B, Hong LL, Carly HM and Acheson AG: Efficacy of transversus abdominis plane blocks in laparoscopic colorectal resections. Surg Endosc 24: 2480-2484, 2010.

8. Brogi E, Cyr S, Kazan R, Giunta F and Hemmerling TM: Clinical performance and pafety of closed-loop systems: A systematic review and meta-analysis of randomized controlled trials. Anesth Analg 124: 446-455, 2017.

9. Dumans-Nizard V, Le Guen M, Sage E, Chazot T, Fischler M and Liu N: Thoracic epidural analgesia with levobupivacaine reduces remifentanil and propofol consumption evaluated by closed-loop titration guided by the bispectral index: A double-blind placebo-controlled study. Anesth Analg 125: 635-642, 2017.

10. Liu Y, Li M, Yang D, Zhang X, Wu A, Yao S, Xue Z and Yue Y: Closed-loop control better than open-loop control of profofol TCI guided by BIS: A randomized, controlled, multicenter clinical trial to evaluate the CONCERT-CL closed-loop system. PLOS One 10: e0123862, 2015.

11. Marsh B, White M, Morton N and Kenny GN: Pharmacokinetic model driven infusion of propofol in children. Br J Anaesth 67: 41-48, 1991.

12. Minto CF, Schnider TW, Egan TD, Youngs E, Lemmens HJ, Gambus PL, Billard V, Hoke JF, Moore KH, Hermann DJ, et al: Influence of age and gender on the pharmacokinetics and pharmacodynamics of remifentanil. I. model development. Anesthesiology 86: 10-23, 1997.
13. Hebbard P,Fujiwara Y, Shibata Y and Royse C: Ultrasound-guided transversus abdominis plane (TAP) block. Anaesth Intensive Care 35: 616-617, 2007.

14. Liu N, Chazot T, Hamada S, Landais A, Boichut N, Dussaussoy C, Trillat B, Beydon L, Samain E, Sessler DI and Fischler M: Closed-loop coadministration of propofol and remifentanil guided by bispectral index: A randomized multicenter study. Anesth Analg 112: 546-557, 2011.

15. Baldini G and Fawcett WJ: Anesthesia for colorectal surgery. Anesthesiol Clin 33: 93-123, 2015.

16. Kumar K, Kirksey MA, Duong S and Wu CL: A Review of opioid-sparing modalities in perioperative pain management: Methods to decrease opioid use postoperatively. Anesth Analg 125: 1749-1960, 2017.

17. Sarin A, Litonius ES, Naidu R, Yost CS, Varma MG and Chen LL: Successful implementation of an enhanced recovery after surgery program shortens length of stay and improves postoperative pain, and bowel and bladder function after colorectal surgery. BMC Anesthesiol 16: 55, 2016.

18. Rafi AN: Abdominal field block: A new approach via the lumbar triangle. Anaesthesia 56: 1024-1026, 2001.

19. Abdallah FW, Laffey JG, Halpern SH and Brull R: Duration of analgesic effectiveness after the posterior and lateral transversus abdominis plane block techniques for transverse lower abdominal incisions: A meta-analysis. Br J Anaesth 111: 721-735, 2013.

20. Johns N, O'Neill S, Ventham NT, Barron F, Brady RR and Daniel T: Clinical effectiveness of transversus abdominis plane (TAP) block in abdominal surgery: A systematic review and meta-analysis. Colorectal Dis 14: 635-642, 2012.

21. Abdallah FW, Chan VW and Brull R: Transversus abdominis plane block: The effects of surgery, dosing, technique, and timing on analgesic outcomes. A systematic review. Reg Anesth Pain Med 37: 193-209, 2012.

22. Eslamian L, Jalili Z, Jamal A, Marsoosi V and Movafegh A: Transversus abdominis plane block reduces postoperative pain intensity and analgesic consumption in elective cesarean delivery under general anaesthesia. J Anesth 26: 334-338, 2012.

23. Pedrazzani C, Menestrina N, Moro M, Brazzo G, Mantovani G, Polaiti E and Guqlielmi A: Local wound infiltration plus transversus abdominis plane (TAP) block versus local wound infiltration in laparoscopic colorectal surgery and ERAS program. Surg Endosc 30: 5117-5125, 2016.

24. Siddiqui MR, Sajid MS, Uncles DR, Cheek L and Baig MK: A meta-analysis of the clinical effectiveness of transverse abdominis plane block. J Clin Anesth 23: 7-14, 2011

25. Guignard B, Menigaux C, Dupont X, Fletcher D and Chauvin M: The effect of remifentanil on the Bispectral index change and hemodynamic responses after orotracheal intubation. Anesth Analg 90: 161-167, 2000.

26. Tsuchiya M, Sato EF, Inoue M and Asada A: Open abdominal surgery increases intraoperative oxidative stress: Can it be prevented? Anesth Analg 107: 1946-1952, 2008.

(i) $(2)$ This work is licensed under a Creative Commons Attribution-NonCommercial-NoDerivatives 4.0 International (CC BY-NC-ND 4.0) License. 